\title{
Understanding Gene-Lifestyle Interaction in Obesity: The Role of Mediation versus Moderation
}

\author{
Louis Pérusse $^{a, b}$ Raphaëlle Jacobb,c, d Vicky Drapeaub, d, e Clare Llewellyn $^{f}$ \\ Benoit J. Arsenault $^{d, g} \quad$ Alexandre Bureau $^{\text {h, i }}$ Marie-Ève Labontéb,c \\ Angelo Tremblay ${ }^{a, b}$ Marie-Claude Vohl ${ }^{b, c}$ \\ aDepartment of Kinesiology, Faculty of Medicine, Université Laval, Québec, QC, Canada; ${ }^{b}$ Centre Nutrition, \\ Santé et Société (NUTRISS), Institute of Nutrition and Functional Foods (INAF), Québec, QC, Canada; 'School of \\ Nutrition, Université Laval, Québec, QC, Canada; ${ }^{\mathrm{d} C e n t r e ~ d e ~ R e c h e r c h e ~ d e ~ I ' I n s t i t u t ~ U n i v e r s i t a i r e ~ d e ~ C a r d i o l o g i e ~ e t ~}$ \\ de Pneumologie de Québec, Université Laval, Québec, QC, Canada; 'Department of Physical Education, Faculty \\ of Education, Université Laval, Québec, QC, Canada; ${ }^{f}$ Research Department of Behavioural Science and Health, \\ University College London, London, UK; ${ }^{9}$ Department of Medicine, Faculty of Medicine, Université Laval, Québec, \\ QC, Canada; ' hepartment of Social and Preventive Medicine, Faculty of Medicine, Université Laval, Québec, QC, \\ Canada; 'CERVO Brain Research Center, Centre Intégré Universitaire de Santé et de Services Sociaux de la \\ Capitale-Nationale, Québec, QC, Canada
}

\section{Keywords}

Obesity · Gene-environment interaction · Mediation analysis · Nutrigenetics

\begin{abstract}
Background: Obesity results from complex interactions between genetic susceptibility to weight gain and poor eating and lifestyle behaviors. The approach that has been traditionally used in genetics to investigate gene-environment/ lifestyle interaction in obesity is based on the concept of moderation or effect modification. Another approach called mediation analysis can be used to investigate gene-environment interaction in obesity. The objective of this review article is to explain the differences between the concepts of moderation and mediation and summarize the studies that have used mediation analysis to support the role of eating or lifestyle behaviors as putative mediators of genetic susceptibility to obesity. Summary: Moderation is used to determine whether the effect of an exposure (genes associated with obesity) on an outcome (obesity phenotype) differs in
\end{abstract}

magnitude and/or direction across the spectrum of environmental exposure. Mediation analysis is used to assess the extent to which the effect of the exposure on the outcome is explained by a given set of hypothesized mediators with the aim of understanding how the exposure could lead to the outcome. In comparison with moderation, relatively few studies used mediation analyses to investigate gene-environment interaction in obesity. Most studies found evidence that traits related to appetite or eating behaviors partly mediated genetic susceptibility to obesity in either children or adults. Key Messages: Moderation and mediation represent two complementary approaches to investigate gene-environment interaction in obesity and address different research questions pertaining to the cause-effect relationship between genetic susceptibility to obesity and various obesity outcomes. More studies relying on mediation are needed to better understand the role of eating and lifestyle habits in mediating genetic susceptibility to obesity.

(c) 2022 The Author(s).

Published by S. Karger AG, Basel
Karger@karger.com www.karger.com/lfg

Karger $\stackrel{\text { ' }}{5}$

BOPEN ACCESS
(C) 2022 The Author(s)

Published by S. Karger AG, Basel

This is an Open Access article licensed under the Creative Common Attribution-NonCommercial-4.0 International License (CC BY-NC) (http://www.karger.com/Services/OpenAccessLicense), applicable to the online version of the article only. Usage and distribution for commercial purposes requires written permission.
Correspondence to:

Louis Pérusse, louis.perusse@ kin.ulaval.ca 


\section{Introduction}

Recent data on the worldwide trends of body mass index (BMI) indicate that the prevalence of obesity (BMI $\geq 30 \mathrm{~kg} / \mathrm{m}^{2}$ for adults and more than 2 SD above the median of the WHO growth reference for children and adolescents) has risen considerably in the past 4 decades [ 1 , 2]. In 2016, an estimated 671 million adults and 124 million children and adolescents were living with obesity compared to 100 million and 11 million, respectively, in 1975. Our obesogenic environment characterized by unhealthy eating habits and a sedentary lifestyle is considered a key driver of this rapid increase in the prevalence of obesity. However, the fact that not everybody develops overweight or obesity despite being exposed to the same obesogenic environment suggests that obesity results from a complex interaction between genetic and environmental factors leading to excessive weight gain in genetically susceptible individuals. The approach traditionally used to investigate gene-environment/lifestyle interactions in obesity has been an examination of the effect of genes (exposure) on obesity (outcome) in groups of individuals stratified based on an environmental factor (e.g., active vs. inactive individuals). In this approach, the environment acts as a moderator of the relationship between the exposure and the outcome. An alternative to provide insight into interplay between genetic and environmental factors in obesity is to use mediation analysis, an approach that has not been frequently used in this context. Unlike moderation, mediation analysis is used to assess the extent to which the effect of an exposure (genes associated with obesity) on an outcome (obesity phenotype) is explained by a given set of hypothesized mediators (also called intermediate variables). Despite extensive evidence supporting the existence of gene-environment interaction in obesity [3-7], few studies have used mediation analysis to identify potential mediators of genetic susceptibility to obesity. The aim of this short review is to (1) explain the differences between the concepts of moderation and mediation and (2) provide a brief overview of the studies that have used mediation analysis to support the role of eating or lifestyle behaviors as putative mediators of genetic susceptibility to obesity.

\section{Concepts of Moderation versus Mediation}

This section provides a brief overview of moderation and mediation and highlights the differences between the two concepts. In-depth reviews [8-10] and full book-

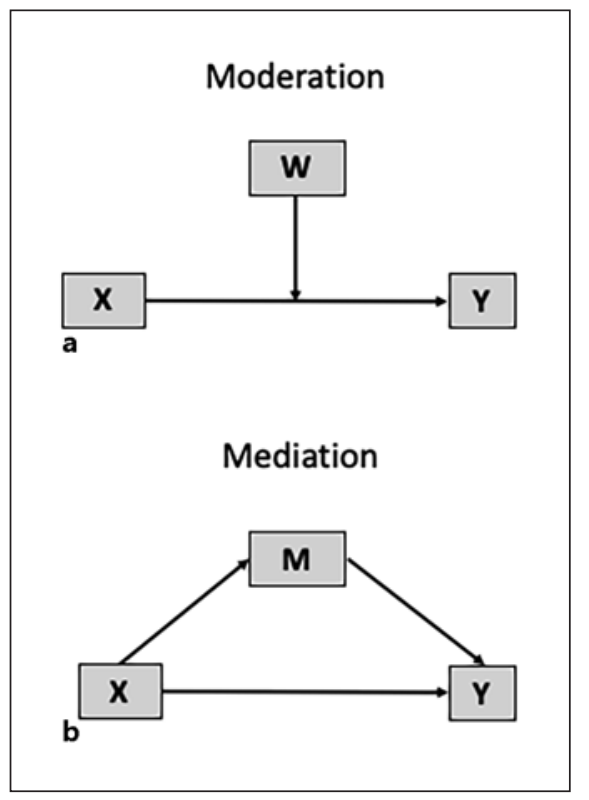

Fig. 1. Basic moderation and mediation models. a Moderation model with a single moderator $\mathrm{W}$ influencing the effect of $\mathrm{X}$ on $\mathrm{Y}$. b Mediation model with a single mediator $M$ causally related between $\mathrm{X}$ and $\mathrm{Y}$.

length overviews $[11,12]$ of these concepts are available elsewhere. Moderation and mediation are used to provide insight into the explanation of causal phenomena, i.e., how exposure affects an outcome [12]. In the context of this review, the exposure $(\mathrm{X})$ represents genetic susceptibility to obesity, while the outcome (Y) represents an obesity phenotype, such as BMI. Although moderation and mediation are interdependent notions and even though researchers tend to use both terms interchangeably, these concepts are different and address different research aims pertaining to the exposure-outcome relationship. The difference between the two concepts is illustrated by the two basic moderation and mediation models illustrated in Figure 1. The concept of moderation is depicted in Figure 1a. The arrow linking $\mathrm{W}$ (the moderator) to the effect of X on Y denotes that X's effect on Y varies according to $\mathrm{W}$ (effect modification). Moderation analysis is thus used to address the $3 \mathrm{Ws}$ of the relationship between $\mathrm{X}$ and $\mathrm{Y}$, i.e., When, or under What circumstances or for Who (what type of people) the effect of X on Y exists or not and in what magnitude [10]. For example, the effect of a genetic variant on BMI may vary by age (When), type of diet (What), or by sex (Who). In the genetic literature, moderation is often referred to as interaction and represents the approach that has been traditionally used to provide
Pérusse et al. 
Fig. 2. Simple mediation model of genetic susceptibility to obesity. Illustration of a simple mediation model in which genetic susceptibility to obesity (independent variable $X$ ) influences an obesity trait (dependent variable $Y$ ) directly $\left(c^{\prime}\right)$ or indirectly through a mediator $M$. The direct and indirect effects add to yield the total effect $(c)$ of $X$ on $Y$. The paths of the model labeled $a, b, c$, and $c^{\prime}$ are estimated using the three regression equations represented in the figure. The indirect effect or mediated effect of $X$ on $Y$ through $M$ is quantified as the product of $a$ and $b(a b)$.

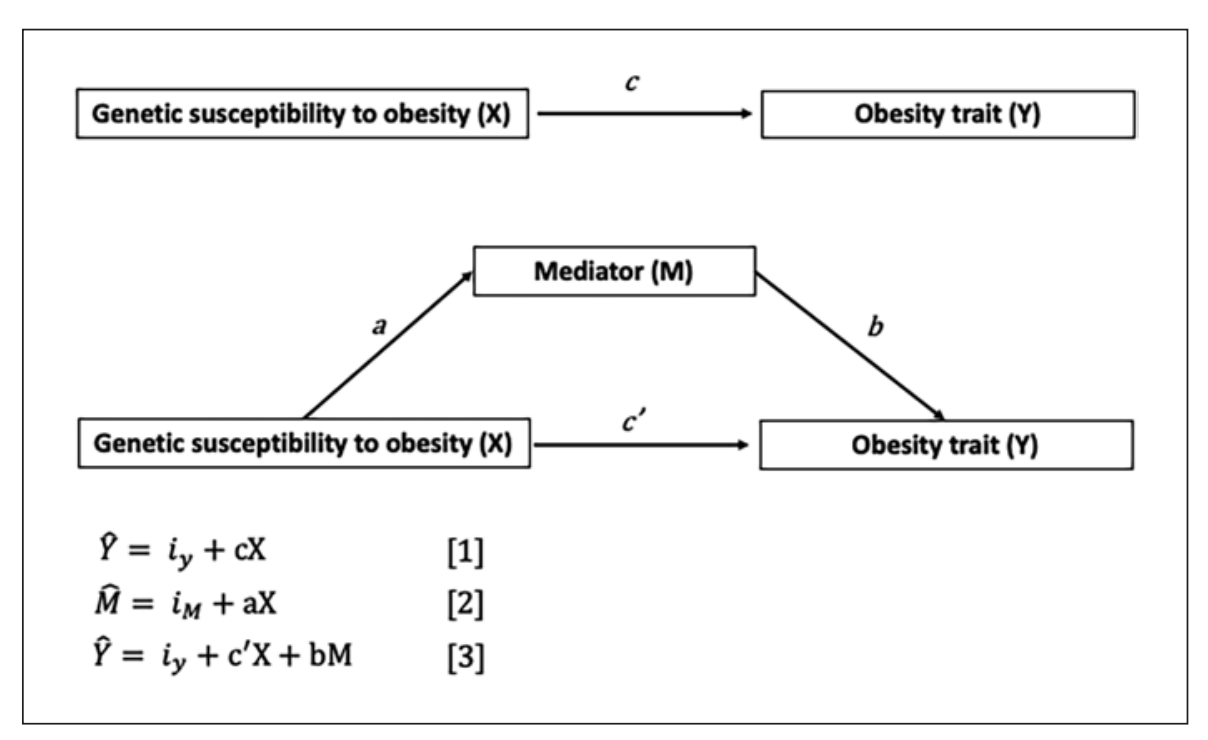

evidence of gene-environment interaction for obesity in observational studies. For example, several recent studies provided evidence that genetic susceptibility to obesity is modified by various lifestyle characteristics such as alcohol consumption [13, 14], sugar-sweetened beverage [15], dietary fat and total energy intake (EI) [16], healthy diet [17, 18], television viewing [19], physical activity [14, 20,21], and sleeping habits [22].

Mediation analysis is used when the aim of the research is to determine How X exerts its effect on Y. In its simple form, mediation is depicted in Figure $1 \mathrm{~b}$ where a mediator M (also called an intermediate variable) is located causally between $\mathrm{X}$ and $\mathrm{Y}$. In such a model there are two pathways by which $\mathrm{X}$ can impact $\mathrm{Y}$. One pathway leads from $\mathrm{X}$ to $\mathrm{Y}$ without passing through $\mathrm{M}$ and is called the direct effect of $\mathrm{X}$ on $\mathrm{Y}$. The second pathway from $\mathrm{X}$ to $\mathrm{Y}$ is the indirect effect of $\mathrm{X}$ on $\mathrm{Y}$ through $\mathrm{M}$; it first passes from $\mathrm{X}$ to $\mathrm{M}$ and then from $\mathrm{M}$ to $\mathrm{Y}$. The indirect effect represents how $\mathrm{Y}$ is influenced by $\mathrm{X}$ through a causal sequence in which $\mathrm{X}$ influences $\mathrm{M}$, which in turn influences Y. Thus, a mediation model is a set of two or more causal events chained together in a sequence of the form $X \rightarrow M$ $\rightarrow \mathrm{Y}[10,11]$. Although mediation analysis has been around for more than 70 years [10], its use was popularized in the late 1980s by Baron and Kenny [23] using an analytic approach based on regression analyses.

In the context of genetic studies of obesity, the objective of mediation analysis is thus to determine the extent to which the relationship between $\mathrm{X}$ (here, single genetic variant or multiple variants associated with obesity) and $\mathrm{Y}$ (here, a measure of obesity) is explained by a hypothe- sized mediator M (or a set of mediators). This objective can be achieved by estimating the regression coefficients of the three regression models represented in the form of the path diagram shown in Figure 2 [10]. The first regression model represents the total effect of X on Y (path $c$, equation [1]). The second regression model represents the effect of X on M (path $a$, equation [2]). The third regression model represents the association between $\mathrm{M}$ and $\mathrm{Y}$ after adjustment for X (path $b$, equation [3]). The effect of X on Y when $\mathrm{M}$ is held constant (path $c^{\prime}$. equation [3]) is called the direct effect (not via the mediator). The effects represented by the regression coefficients in equations [1-3] can be estimated with any regression analysis or structural equation modeling program $[24,25]$. A computational tool for path analysis-based moderation and mediation analyses called PROCESS (for SPSS, SAS, and R) has been developed for estimating direct and indirect effects for various mediation models, allowing for one or multiple mediators simultaneously or for the presence of an interaction between the mediators and the exposure $[10,11]$. The indirect effect of $\mathrm{X}$ on $\mathrm{Y}$ through mediator $\mathrm{M}$ quantifies the estimated difference in $\mathrm{Y}$ resulting from one-unit change in $\mathrm{X}$ through the causal sequence $\mathrm{X} \rightarrow \mathrm{M} \rightarrow \mathrm{Y}$ [10]. This indirect effect represents the amount of mediation and is estimated as the product of regression coefficients $a$ and $b$ in equations $[2,3]$. Thus, in a mediation model, the total effect equals the sum of direct and indirect effects $\left(c=c^{\prime}+a b\right)$ and the indirect effect equals the reduction of the effect of $\mathrm{X}$ on $\mathrm{Y}$ when $\mathrm{M}$ is controlled versus when it is not $\left(a b=c-c^{\prime}\right)$. The indirect effect divided by the total effect $(a b / c)$ can also be 
computed to obtain a measure of the proportion of the total effect that is mediated, A rejection of the null hypothesis that the indirect effect is zero $(a b=0)$ is sufficient to support a claim of mediation of the effect of X on $\mathrm{Y}$ through M [10]. Various approaches have been proposed to test this null hypothesis. One of the earlier and very popular tests that was used to infer about mediation is the Sobel test [26]. However, the test is considered low in power because it assumes that the sampling distribution of $a b$ is normal, which is rarely the case [27]. Therefore, alternative approaches performing better than the Sobel test without making any assumptions about the sampling distribution of $a b$ have been proposed [10, 27]. Among these methods, the bootstrap confidence interval (CI) method is considered a good compromise balancing validity and power considerations. Bootstrapping is a nonparametric method of inference based on resampling methods. Briefly, the original sample size $n$ is treated as a miniature representation of the population originally sampled. The observations in the sample are then resampled with replacement and the statistic of interest is computed in the new sample constructed through this resampling process. The procedure is repeated multiple times, thousands of times ideally, leading to an empirical sampling distribution of the statistic of interest. In mediation analysis, the method is used to generate a sampling distribution of the indirect effect $a b$, which can be used to derive $95 \%$ CIs for the indirect effect. If the 95\% $\mathrm{CI}$ is entirely above or below zero, this supports a claim of mediation, whereas a CI straddling zero indicates that data are compatible with the absence of mediation. The Sobel test and the bootstrap CI method are both implemented in PROCESS macros for SPSS, SAS, and R.

In summary, moderation and mediation analyses address different research questions pertaining to the exposure-outcome relationship $[9,12]$ and can be viewed as complementary approaches to investigate gene-environment interaction in obesity. Moderation analysis is used to investigate the role of environment/lifestyle in modifying the association between the genetic susceptibility to obesity (exposure) and a trait associated with obesity (outcome). Mediation analysis is used to identify environmental/lifestyle factors explaining how genetic susceptibility exerts its effects on an obesity outcome. Assessing interaction/moderation may help identify a subgroup of the population who may benefit the most from an intervention. For example, if the impact of genetic susceptibility to obesity on body weight is much greater in a subgroup of the population, e.g., in inactive versus active individuals, then it might be more appropriate to intervene in inactive individuals first instead of intervening on the entire population. Mediation is mainly motivated by the wish to understand the pathways whereby an exposure leads to the outcome with the aim of intervening on the mediator to reduce the risk of disease or improve the outcome. For example, if the effect of genetic susceptibility to obesity on body weight is mainly mediated by eating behavior (EB) traits such as disinhibition (DIS) or satiety responsiveness (SR), then refining the intervention further to target these traits may lead to a more favorable outcome in an intervention trial.

Finally, it is worth noting that both moderation and mediation can occur simultaneously to explain the relationship between the exposure and the outcome. Taking the FTO gene as example and considering the evidence showing that the effect of FTO on obesity is modified by physical activity level (PAL) [28] and that PAL acts as a mediator of the association between FTO and obesity [29], we can postulate that the effect of FTO on obesity may operate through both mediation and moderation (interaction). In that context, PAL would act as a mediator of the association between FTO and obesity, but in inactive individuals only, for example. To the best of our knowledge, no studies have investigated the relative impact of mediation versus moderation in explaining the effect of genetic susceptibility to obesity, even though the methodology described above to assess mediation has been extended to allow for the presence of a mediator interacting with the exposure $[30,31]$. In that context, the overall effect of the exposure on the outcome can be decomposed into components due to just mediation, to just interaction, to both mediation and interaction, and to neither mediation nor interaction. This four-way decomposition model, which unifies methods to assess effects due to interaction and methods to assess mediation [31], has been implemented in a SAS procedure called CAUSALMED [32]. Future studies based on this unified approach will contribute to improve our understanding of the interplay between genetic and lifestyle factors in the development of obesity.

\section{Role of Eating and Lifestyle Behaviors in Mediating Genetic Susceptibility to Obesity}

This section presents an overview of the studies that have used mediation analyses to identify eating or lifestyle behaviors mediating genetic susceptibility to obesity. These studies are summarized in Table 1. Most of the studies were based on the behavioral susceptibility theory of obesity, which proposes that genetic susceptibility to obesity is partly attributable to appetite regulation and 


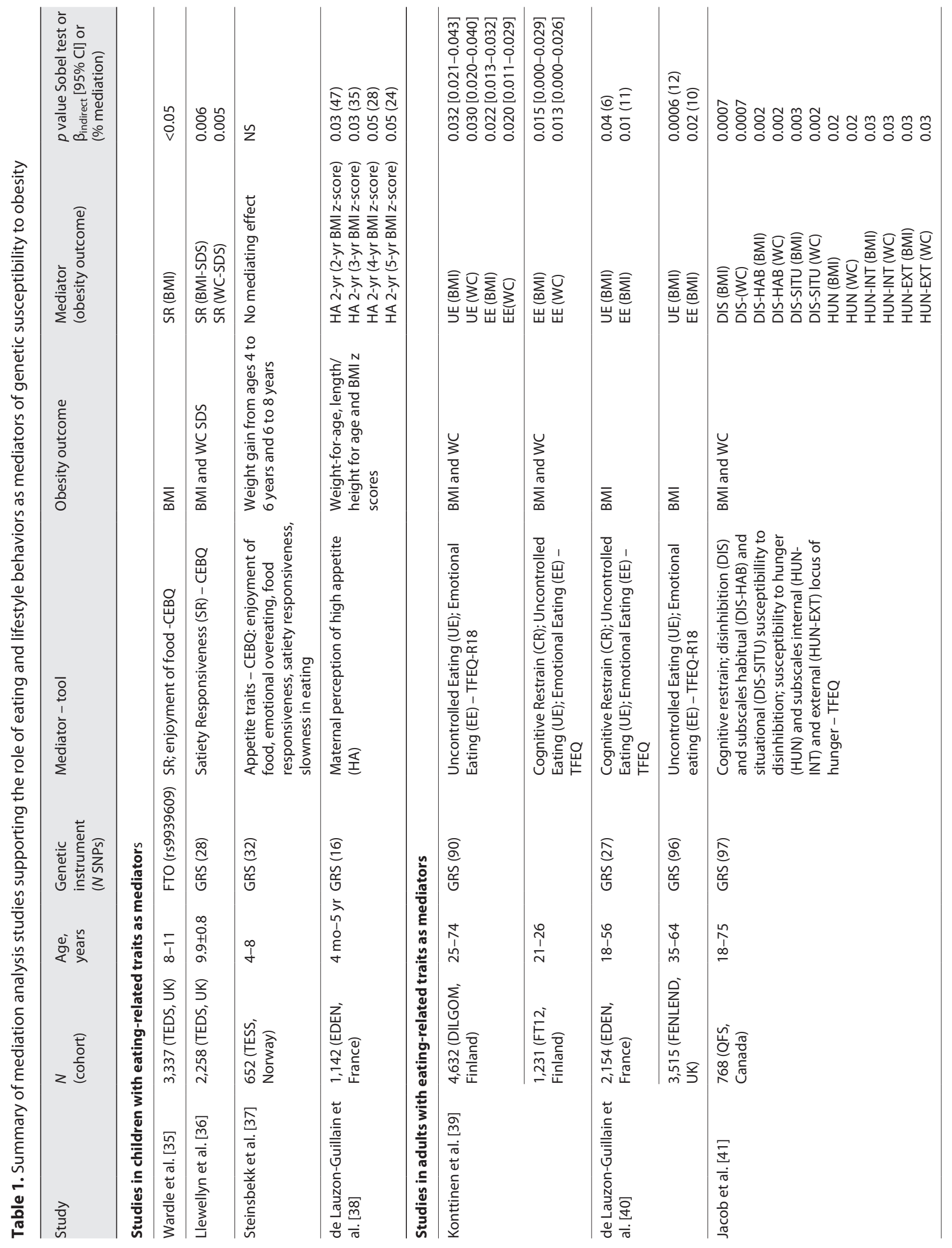




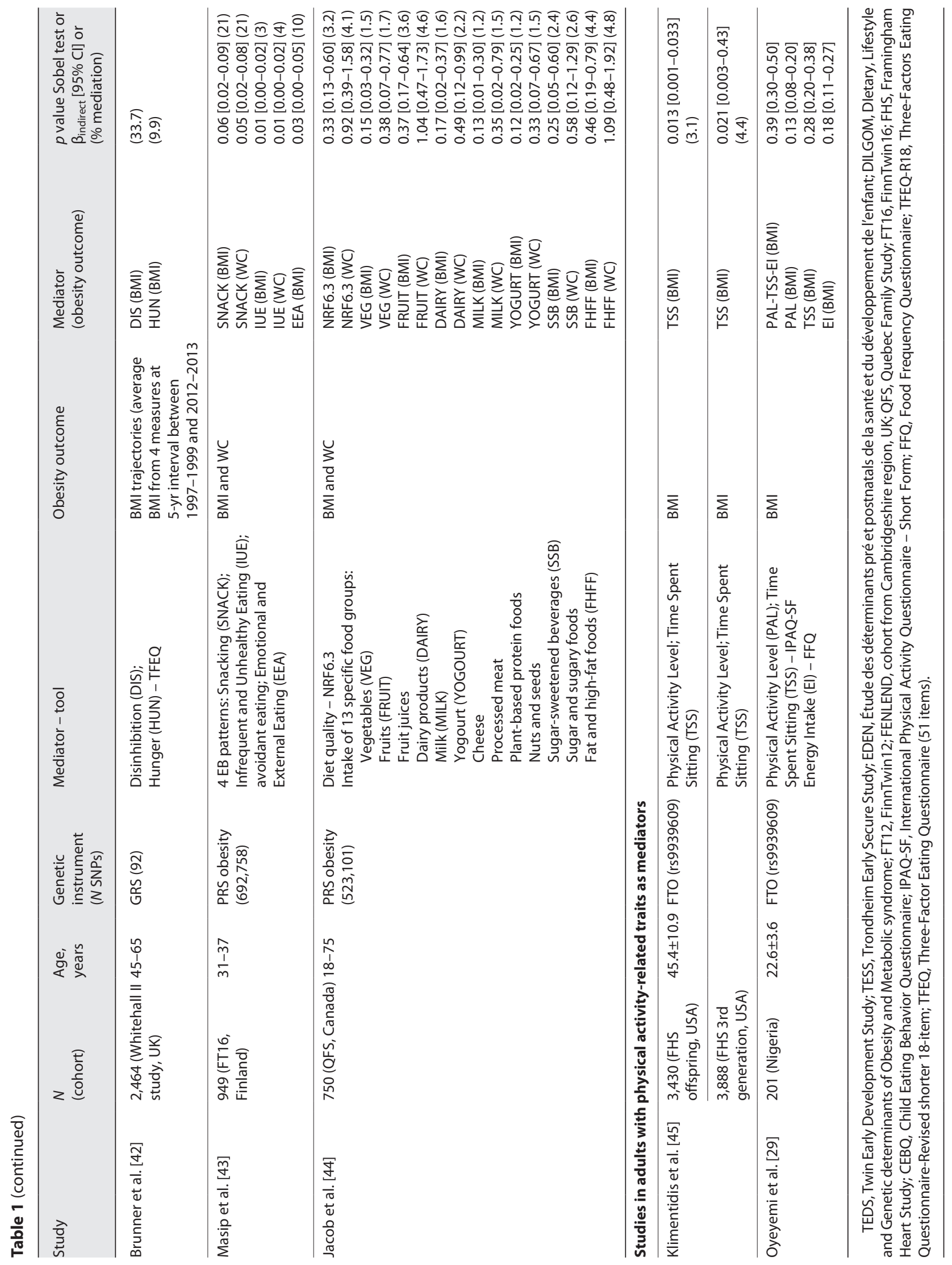


that $\mathrm{EB}$ traits are the behavioral expression of genetic risk of obesity [33]. Several studies reviewed elsewhere [34] investigated the role of EB traits in genetic susceptibility to obesity. Most of the mediation studies summarized in Table 1 investigated the role of eating-related traits as putative mediators of genetic susceptibility to obesity in children or adults and provided support to the behavioral susceptibility theory. The first study was conducted on a sample of 3,337 children aged 8-11 years from the Twin's Early Development Study (TEDS) [35]. The role of two appetite-related traits, SR, a parent-report measure of child appetite, and enjoyment of food, a measure of the extent to which presentation of palatable foods provokes eating, as mediators of the effect of FTO rs9939609 single nucleotide polymorphism (SNP) on measures of BMI and waist circumference taken by the parents was investigated. Results indicated that the effect of the FTO genotype on BMI was partially mediated by SR. A follow-up of this first study undertaken in 2,258 ten-year-old children showed that SR mediated the association between a genetic risk score (GRS) of obesity comprising 28-BMI SNPs and standardized scores (SDS) of BMI $(p=0.006)$ and waist circumference ( $p=0.005)$ [36]. Two other studies investigated the mediating role of appetite-related traits on the genetic susceptibility to obesity assessed in children. The first one examined the association between a GRS of obesity based on 32 SNPs and 5 EB traits measured with the Child EB Questionnaire in 652 children aged 4-8 years [37]. Although results indicated that children with a higher GRS gained weight more rapidly and have a faster eating rate, none of the EB traits significantly mediated the association with weight gain. In the second study, the association between a BMI-GRS of obesity based on 16 SNPs associated with childhood BMI $z$-scores and mother-reported EB in children up to 5 years of age was examined in 1,142 children from the Étude des Déterminants pré et postnatals de la santé de l'Enfant (EDEN) birth cohort [38]. High maternal perception of their child's appetite at 2 years of age was found to partly mediate the association between the BMI-GRS and BMI $\mathrm{z}$-scores with a mediation ratio decreasing from $47 \%$ to $24 \%$ between the ages 2 and 5 .

The role of eating-related traits in mediating genetic susceptibility to obesity has also been investigated in adults. In the first study based on two independent population-based Finish cohorts, the Dietary, lifestyle and genetic determinants of obesity and metabolic syndrome Study (4,632 individuals aged $25-74$ years) and the FinnTwin 12 Study (1,231 twins aged 21-26 years), the association between a GRS of obesity assessed from 90 BMI-

Mediation Analysis in the Study of Gene-Lifestyle Interaction in Obesity associated SNPs and measures of BMI and waist circumference was found to be partly mediated through higher levels of uncontrolled eating and emotional eating [39]. A second study replicated these findings using data from two cohorts of adults in France (EDEN cohort) and in the UK (Fenland cohort) [40]. Results showed that both emotional eating and uncontrolled eating mediated the association between a GRS of obesity (96 SNPs for Fenland and 27 SNPs for EDEN) and BMI. In a third study based on 768 adults from the Quebec Family Study (QFS) [41], we showed that individuals at greater genetic risk of obesity (based on 97 BMI-associated SNPs) reported more habitual and situational disinhibited eating and a greater tendency to feel hungry in response to both internal and external cues. These EB traits partly mediated the association between genetic susceptibility to obesity and BMI and waist circumference measures [41]. A fourth study investigated the mediating role of EB in genetic susceptibility to weight gain in 2,464 British adults aged 45-65 years who had repeated measurements of BMI on four occasions at 5-year intervals over 20 years [42]. Results revealed a significant mediating role of DIS and hunger accounting for $33.7 \%$ and $9.9 \%$, respectively, of the relationship between genetic susceptibility to obesity and average BMI measurements from 45 to 65 years. There was no significant increase in the mediating effect in a model incorporating both mediators (\% mediation 33.9\%), suggesting that in this sample the mediating effect was driven mostly by DIS rather than hunger [42].

Two recent studies undertaken in adults used a more powerful approach to assess genetic susceptibility to obesity by computing a polygenic risk score (PRS) of obesity incorporating whole-genome-based SNPs irrespective of their genome-wide significance. In the first study undertaken in the FinnTwin 16 cohort of Finnish twins, four EB patterns identified using principal component analysis and a score of diet quality based on a short food frequency questionnaire, were tested for their role in mediating the association between genetic susceptibility to obesity and BMI $(n=949)$ and waist circumference $(n=874)$ [43]. Among the four EB patterns tested, only the snacking and the infrequent and unhealthy EB patterns mediated the association between the obesity PRS and both measures of obesity. The emotional and external EB pattern only mediated the association of the PRS with BMI [43]. When tested without referring to infrequent eating, the diet quality score alone did not mediate the association between the PRS and obesity [43]. In a second recent study undertaken in 750 participants from QFS, we investigated the mediating effects of diet quality and intake of 
13 specific food groups on the association between genetic susceptibility to obesity and BMI and waist circumference [44]. Dietary intakes were assessed using a 3-day food record from which a diet quality score (the Nutrient Rich Food Index 6.3) and food groups were derived. We found that the association between the obesity PRS and BMI was partly mediated by poor diet quality, high intakes of fat and high-fat foods and sugar-sweetened beverages, and low intakes of vegetables, fruits, and dairy products. Similar trends were observed for waist circumference [44].

Finally, only two studies investigated the mediating role of traits related to PAL in mediating genetic susceptibility to obesity. The first study based on 7,318 individuals from the Offspring and third-generation cohorts of the Framingham Heart Study (FHS) examined possible mediation effects of PAL and time spent sitting (TSS, as an indicator of physical inactivity) on the association between the FTO rs9939609 SNP and BMI [45]. Mediation analyses revealed that the association between the FTO genotype and BMI was partly mediated by TSS (\%mediation $~ 3 \%-4 \%$ ), but not by PAL. In another study, the role of PAL, TSS, and EI as putative mediators of the relationship between FTO rs9939609 SNP and BMI was examined in 201 young adults from the Nigerian population [29]. Mediation analyses revealed a combined mediating effect of PAL, TSS, and EI in the association between FTO genotype and BMI ( $\beta_{\text {indirect }}=0.39$; 95\% CI: $\left.0.30-0.50\right)$. Analyses also revealed a significant mediating effect of each mediator tested separately [29]. These studies were limited by their use of only one FTO SNP to assess genetic susceptibility to obesity.

One assumption of mediation analysis common to the studies reviewed above is that the alleged mediator is causally involved in the pathway linking the exposure to the outcome, which also implies that the mediator must precede the effect. Causality and temporality are thus important limitations of mediation analyses that have been published so far. These limitations can be addressed by performing Mendelian randomization to identify eating or lifestyle mediators causally related to obesity before undertaking mediation analysis. The assessment of genetic susceptibility to obesity based on a relatively small number of SNPs associated with obesity represents another limitation of most studies undertaken so far. The use of PRSs incorporating whole-genome-based SNPs irrespective of their genome-wide significance represents a more powerful approach to estimate of an individual's genetic susceptibility to a trait and should be considered in future studies.

\section{Summary and Conclusion}

Moderation and mediation represent two complementary approaches to investigate gene-environment interaction in obesity or the cause-effect relationship between genetic susceptibility to obesity and various obesity outcomes. One way to investigate this cause-effect relationship is to explain When, for Whom, and in What circumstances the cause influences the outcome and this refers to the concept of moderation. Another way to investigate this cause-effect relationship is to explain How the effect occurs and this refers to the concept of mediation. Both approaches can help refine weight loss and maintenance programs. In the case of moderation, it may help identify a subgroup of the population for whom the intervention is likely to benefit the most. In the case of mediation, it may help to design intervention programs that will target a mediator causally related to the outcome, which could potentially increase impact of the intervention on the outcome. So far, gene-environment interactions in obesity have mainly been investigated through moderation. Future studies relying on mediation and allowing for both moderation and mediation will contribute to improve our understanding of the role of eating and lifestyle habits in explaining how genetic susceptibility to obesity impacts body weight or other obesity-related traits.

\section{Conflict of Interest Statement}

Louis Pérusse is Associate Editor of Lifestyle Genomics. Raphaëlle Jacob, Vicky Drapeau, Clare Llewellyn, Benoît J. Arsenault, Alexandre Bureau, Marie-Ėve Labonté, Angelo Tremblay, and Marie-Claude Vohl have no conflict of interest to disclose.

\section{Funding Sources}

Louis Pérusse, Raphaëlle Jacob, Vicky Drapeau, Clare Llewellyn, Benoît J. Arsenault, Alexandre Bureau, Marie-Ève Labonté, Angelo Tremblay, and Marie-Claude Vohl are supported by a grant from the Canadian Institutes for Health Research (PJT-178209). Marie-Claude Vohl is Canada Research Chair in Genomics Applied to Nutrition and Metabolic Health.

\section{Author Contributions}

Louis Pérusse was responsible for conceptualizing, writing, and revising the article. Raphaëlle Jacob, Vicky Drapeau, Clare Llewellyn, Benoît J. Arsenault, Alexandre Bureau, Marie-Ève Labonté, Angelo Tremblay, and Marie-Claude Vohl read, edited and approved the final manuscript.
Pérusse et al. 


\section{References}

1 NCD Risk Factor Collaboration (NCDRisC). Trends in adult body-mass index in 200 countries from 1975 to 2014: a pooled analysis of 1698 population-based measurement studies with 19.2 million participants. Lancet. 2016;387(10026):1377-96.

2 NCD Risk Factor Collaboration (NCDRisC). Worldwide trends in body-mass index, underweight, overweight, and obesity from 1975 to 2016: a pooled analysis of 2416 population-based measurement studies in 128.9 million children, adolescents, and adults. Lancet. 2017;390(10113):262742.

3 Bouchard C. Gene-environment interactions in the etiology of obesity: defining the fundamentals. Obesity. 2008;16(Suppl 3): S5-10.

4 Garver WS, Newman SB, Gonzales-Pacheco DM, Castillo JJ, Jelinek D, Heidenreich RA, et al. The genetics of childhood obesity and interaction with dietary macronutrients. Genes Nutr. 2013;8(3):271-87.

5 Huang T, Hu FB. Gene-environment interactions and obesity: recent developments and future directions. BMC Med Genomics. 2015;8 Suppl 1(Suppl 1):S2.

6 Reddon H, Guéant JL, Meyre D. The importance of gene-environment interactions in human obesity. Clin Sci. 2016;130(18): 1571-97.

7 Sulc J, Mounier N, Gunther F, Winkler T, Wood AR, Frayling TM, et al. Quantification of the overall contribution of gene-environment interaction for obesity-related traits. Nat Commun. 2020;11(1):1385.

8 VanderWeele TJ. Mediation analysis: a practitioner's guide. Annu Rev Public Health. 2016;37:17-32.

9 Corraini P, Olsen M, Pedersen L, Dekkers OM, Vandenbroucke JP. Effect modification, interaction and mediation: an overview of theoretical insights for clinical investigators. Clin Epidemiol. 2017;9:331-8.

10 Hayes AF, Rockwood NJ. Regression-based statistical mediation and moderation analysis in clinical research: observations, recommendations, and implementation. Behav Res Ther. 2017;98:39-57.

11 Hayes AF. Introduction to mediation, moderation and conditional process analysis: a regression-based approach. In: Kenny DA, Little TD, editors. New York: Guilford Press; 2018.

12 VanderWeele TJ. Explanation in causal inference: methods for mediation and interaction. Oxford University Press; 2015. p. 706.

13 Nakamura S, Narimatsu H, Sato H, Sho R, Otani K, Kawasaki R, et al. Gene-environment interactions in obesity: implication for future applications in preventive medicine. J Hum Genet. 2016;61(4):317-22.
14 Rask-Andersen M, Karlsson T, Ek WE, Johansson $\AA$. Gene-environment interaction study for BMI reveals interactions between genetic factors and physical activity, alcohol consumption and socioeconomic status. PLoS Genet. 2017;13(9):e1006977.

15 Qi Q, Chu AY, Kang JH, Jensen MK, Curhan GC, Pasquale LR, et al. Sugarsweetened beverages and genetic risk of obesity. N Engl J Med. 2012;367(15):138796.

16 Celis-Morales CA, Lyall DM, Gray SR, Steell L, Anderson J, Iliodromiti S, et al. Dietary fat and total energy intake modifies the association of genetic profile risk score on obesity: evidence from 48170 UK biobank participants. Int J Obes. 2017;41(12): 1761-8.

17 Ding M, Ellervik C, Huang T, Jensen MK, Curhan GC, Pasquale LR, et al. Diet quality and genetic association with body mass index: results from 3 observational studies. Am J Clin Nutr. 2018;108(6):1291-300.

18 Wang T, Heianza Y, Sun D, Huang T, Ma W, Rimm EB, et al. Improving adherence to healthy dietary patterns, genetic risk, and long term weight gain: gene-diet interaction analysis in two prospective cohort studies. BMJ. 2018;360:j5644.

19 Qi Q, Li Y, Chomistek AK, Kang JH, Curhan GC, Pasquale LR, et al. Television watching, leisure time physical activity, and the genetic predisposition in relation to body mass index in women and men. Circulation. 2012;126(15):1821-7.

20 Ahmad S, Rukh G, Varga TV, Ali A, Kurbasic A, Shungin D, et al. Gene x physical activity interactions in obesity: combined analysis of 111,421 individuals of European ancestry. PLoS Genet. 2013;9(7):e1003607.

21 Li S, Zhao JH, Luan J, Ekelund U, Luben $\mathrm{RN}$, Khaw KT, et al. Physical activity attenuates the genetic predisposition to obesity in 20,000 men and women from EPIC-Norfolk prospective population study. PLoS Med. 2010;7(8):e1000332.

22 Celis-Morales C, Lyall DM, Guo Y, Steell L, Llanas D, Ward J, et al. Sleep characteristics modify the association of genetic predisposition with obesity and anthropometric measurements in 119,679 UK biobank participants. Am J Clin Nutr. 2017;105(4): 980-90.

23 Baron RM, Kenny DA. The moderator-mediator variable distinction in social psychological research: conceptual, strategic, and statistical considerations. J Pers Soc Psychol. 1986;51(6):1173-82.

24 Gunzler D, Chen T, Wu P, Zhang H. Introduction to mediation analysis with structural equation modeling. Shanghai Arch Psychiatry. 2013;25(6):390-4.
25 Gunzler D, Morris N, Tu XM. Causal mediation analysis using structure equation models. In: He H, Wu P, Chen DG, editors. Statistical causal inferences and their applications in public health research. ICSA book series in statistics. Switzerland: Springer; 2016. p. 295-314.

26 Sobel ME. Asymptotic confidence intervals for indirect effects in structural equation models. Sociol Methodol. 1982;13:290312 .

27 Hayes AF, Scharkow M. The relative trustworthiness of inferential tests of the indirect effect in statistical mediation analysis: does method really matter? Psychol Sci. 2013;24(10):1918-27.

28 Kilpelainen TO, Qi L, Brage S, Sharp SJ, Sonestedt E, Demerath E, et al. Physical activity attenuates the influence of FTO variants on obesity risk: a meta-analysis of 218,166 adults and 19,268 children. PLoS Med. 2011;8(11):e1001116.

29 Oyeyemi BF, Ologunde CA, Olaoye AB, Alamukii NA. FTO gene associates and interacts with obesity risk, physical activity, energy intake, and time spent sitting: Pilot Study in a Nigerian population. J Obes. 2017;2017:3245270

30 Valeri L, Vanderweele TJ. Mediation analysis allowing for exposure-mediator interactions and causal interpretation: theoretical assumptions and implementation with SAS and SPSS macros. Psychol Methods. 2013; 18(2):137-50.

31 VanderWeele TJ. A unification of mediation and interaction: a 4-way decomposition. Epidemiology. 2014;25(5):749-61.

32 SAS Institute Inc. The CAUSALMED procedure. SAS/STAT 151 user's guide. Cary, NC: SAS Institute Inc.; 2018. p. 2305-68.

33 Llewellyn C, Wardle J. Behavioral susceptibility to obesity: gene-environment interplay in the development of weight. Physiol Behav. 2015;152(Pt B):494-501.

34 Herle M, Smith AD, Kininmonth A, Llewellyn C. The role of eating behaviours in genetic susceptibility to obesity. Curr Obes Rep. 2020;9(4):512-21.

35 Wardle J, Carnell S, Haworth CM, Farooqi IS, O'Rahilly S, Plomin R. Obesity associated genetic variation in FTO is associated with diminished satiety. J Clin Endocrinol Metab. 2008;93(9):3640-3.

36 Llewellyn CH, Trzaskowski M, Plomin R, Wardle J. From modeling to measurement: developmental trends in genetic influence on adiposity in childhood. Obesity. 2014; 22(7):1756-61.

37 Steinsbekk S, Belsky D, Guzey IC, Wardle J, Wichstrøm L. Polygenic risk, appetite traits, and weight gain in middle childhood: a Longitudinal Study. JAMA Pediatr. 2016;170(2):e154472. 
38 de Lauzon-Guillain B, Koudou YA, Botton J, Forhan A, Carles S, Pelloux V, et al. Association between genetic obesity susceptibility and mother-reported eating behaviour in children up to 5 years. Pediatr Obes. 2019;14(5):e12496.

39 Konttinen H, Llewellyn C, Wardle J, Silventoinen $K$, Joensuu A, Mannisto S, et al. Appetitive traits as behavioural pathways in genetic susceptibility to obesity: a population-based cross-sectional study. Sci Rep. 2015;5:14726.

40 de Lauzon-Guillain B, Clifton EA, Day FR, Clement K, Brage S, Forouhi NG, et al. Mediation and modification of genetic susceptibility to obesity by eating behaviors. Am J Clin Nutr. 2017;106(4):996-1004.
41 Jacob R, Drapeau V, Tremblay A, Provencher V, Bouchard C, Pérusse L. The role of eating behavior traits in mediating genetic susceptibility to obesity. Am J Clin Nutr. 2018;108(3):445-52.

42 Brunner EJ, Maruyama K, Shipley M, Cable $\mathrm{N}$, Iso $\mathrm{H}$, Hiyoshi $\mathrm{A}$, et al. Appetite disinhibition rather than hunger explains genetic effects on adult BMI trajectory. Int J Obes. 2021;45(4):758-65.
43 Masip G, Silventoinen K, Keski-Rahkonen A, Palviainen T, Sipila PN, Kaprio J, et al. The genetic architecture of the association between eating behaviors and obesity: combining genetic twin modeling and polygenic risk scores. Am J Clin Nutr. 2020; 112(4):956-66.

44 Jacob R, Bertrand C, Llewellyn C, Couture C, Labonte ME, Tremblay A, et al. Dietary mediators of the genetic susceptibility to obesity-results from the Quebec Family Study. J Nutr. 2022;152(1):49-58.

45 Klimentidis YC, Arora A, Chougule A, Zhou J, Raichlen DA. FTO association and interaction with time spent sitting. Int J Obes. 2016;40(3):411-6. 